\title{
Влияние условий теплоотвода на характеристики концентраторных фотоэлектрических модулей
}

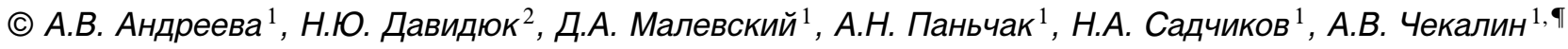 \\ ${ }^{1}$ Физико-технический институт им. А.Ф. Иоффе Российской академии наук, \\ 194021 Санкт-Петербург, Россия \\ ${ }^{2}$ Санкт-Петербугский национальный исследовательский \\ Академический университет Российской академии наук, \\ 194021 Санкт-Петербург, Россия \\ I E-mail: chekalin@mail.ioffe.ru
}

(Получена 12 сентября 2017 г. Принята к печати 26 сентбря 2017 г.)

\begin{abstract}
Приведены результаты исследований влияния различных условий отвода тепла на нагрев и распределение температур в элементах концентраторных фотоэлектрических модулей. Были исследованы модули, выполненные на основе линз Френеля и трехкаскадных фотоэлектрических преобразователей (ФЭП) $\mathrm{InGaP} / \mathrm{GaAs} / \mathrm{Ge}$, установленных на теплосбрасывающие платы, выполненные из меди и стали. Для определения тепловых характеристик использовалась методика, позволяющая измерять температуру $p-n$-переходов ФЭП в лабораторных условиях при имитации тепловых процессов, возникающих при работе модуля в натурных условиях. В дальнейшем по этой температуре рассчитывалась величина теплового сопротивления системы „ФЭП-окружающая среда“. Имитация тепловых процессов в модуле осуществлялась за счет пропускания тока через ФЭП в прямом направлении. Величина разогрева ФЭП определялась путем сопоставления значений прямого падения напряжений, измеренных за время его быстрого включения или выключения при различных условиях отвода тепла. Изменение условий теплоотвода обеспечивалось при помощи генератора воздушного потока.
\end{abstract}

DOI: 10.21883/FTP.2018.03.45627.8725

\section{1. Введение}

Эффективность преобразования солнечного излучения в электроэнергию с помощью концентраторных фотоэлектрических модулей (КФЭМ) сильно зависит от температуры $p-n$-переходов фотоэлектрических преобразователей (ФЭП) в составе модуля $[1,2]$. Основной сложностью при определении зависимостей характеристик модулей от тепловых нагрузок является невозможность непосредственного измерения температуры $p-n$-перехода ФЭП. Наиболее надежным параметром, который может характеризовать температуру $p-n$-перехода, является напряжение холостого хода фотоэлемента $\left(U_{o c}\right)$. Изменение температуры $p-n$-перехода при солнечном освещении может быть определено по разности значений $U_{o c}$, соответствующих рабочему режиму и „холодному“ состоянию, при котором температура ФЭП равна температуре окружающей среды. При этом для определения температуры необходимо знание температурного коэффициента, характеризующего величину изменения $U_{o c}$ при изменении температуры. Наиболее важным является определение температуры при работе модулей в режиме оптимальной нагрузки, когда часть преобразуемой солнечной энергии переносится электрическим током в нагрузку. Сложность этой задачи заключается в том, что для определения $U_{o c}$, соответствующего температуре ФЭП в рабочем режиме, необходимо быстрое переключение системы в режим холостого хода. При этом время измерения $U_{o c}$ при переключении должно быть настолько малым, чтобы температура $p-n$-переходов за время измерения не успела измениться. Еще бо́льшие сложности в натурных условиях возникают при определении значения $U_{o c}$ в „холодном“ состоянии, когда в модуле за короткое время на все затененные фотоэлементы, находящиеся при температуре окружающей среды, должно быть направлено солнечное излучение и измерен скачок напряжения $U_{o c}$, так чтобы температура $p-n$-переходов за время измерения $U_{o c}$ не успела измениться. В работах [3-6] был предложен метод очень быстрого переключения внешней электрической нагрузки в исследуемых модулях с помощью электронного коммутатора. Температурный коэффициент был определен в лабораторных условиях на отдельных ФЭП и в модулях, облучаемых имитатором солнечного излучения для концентраторных модулей [3-5].

Предложенный метод позволяет определять температуру $p-n$-перехода ФЭП в концентраторных фотоэлектрических модулях в любых условиях окружающей среды. Эти условия зачастую не являются постоянными. На температуру модуля оказывают влияние солнечный спектр, интенсивность освещения, скорость и направление ветра. Для сравнения характеристик модулей разной конструкции целесообразно использовать более универсальный параметр - тепловое сопротивление $\left(r_{\text {th }}\right)$ системы „ФЭП-окружающая среда“, позволяющий оценивать эффективность рассеяния тепла модулем в различных условиях внешней среды [7]. Однако условия окружающей среды могут значительно изменяться за время измерений, что вносит существенную погрешность в измерения рабочей температуры фотопреобразователей. К дополнительным ошибкам приводит неравномерность нагрева задней панели модуля с расположенными на ней 
фотоэлементами. Из-за указанных выше погрешностей требуются длительное время для проведения измерений, фильтрация полученных данных и статистическая обработка большого объема результатов, полученных во внешних условиях [7]. Чтобы исключить ошибки, связанные с изменением интенсивности солнечного излучения в натурных условиях, предложена методика, при которой теплофизические свойства КФЭМ определяются в помещении в затемненных условиях [8]. При этой методике температура $p-n$-переходов и тепловое сопротивление определяются при создании тепловой нагрузки модулей путем пропускания прямого тока. Индикатором температуры $p-n$-переходов являются значения прямого падения напряжения, определенные из вольт-амперной характеристики при определенном значении силы тока. В этом случае для определения температуры были определены значения температурного коэффициента $\beta$, характеризующего зависимость величины падения прямого напряжения ФЭП от температуры, путем измерения вольт-амперных характеристик ФЭП или модулей в термостатной камере при разных температурах.

Целью данной работы являлось исследование влияния условий теплосъема, характеризуемых разными направлениями и скоростями обдува модулей, на величину перегрева ФЭП в концентраторных фотоэлектрических модулях. Для этого была использована методика, позволяющая измерять температуру $p-n$-переходов ФЭП и тепловое сопротивление системы „ФЭП-окружающая среда“" в лабораторных условиях [8].

\section{2. Экспериментальные образцы и условия измерений}

Для экспериментов были выбраны концентраторные фотоэлектрические модули SMALFOC [4], разработанные в Физико-техническом институте им. А.Ф. Иоффе. Измерения проводились на модулях размерами $1 \times 0.5$ м, включающих в себя две параллельные панели из силикатного стекла. На передней панели располагались 128 линз Френеля, выполненных из силикона, размером $60 \times 60$ мм, на задней панели в фокусах линз Френеля размещалось соответствующее количество трехкаскадных ФЭП InGaP/GaAs/Ge. Для отвода тепла фотопреобразователи были размещены на металлических теплосбрасывающих платах, на каждой по 8 параллельно соединенных ФЭП. 16 таких плат соединялись между собой последовательно. Теплосбрасывающие платы были изготовлены методом штамповки либо из меди толщиной 0.5 мм, либо из стали толщиной 1 мм и установлены на наружной стороне задней панели так, чтобы сфокусированное пятно солнечного излучения от каждой линзы Френеля совпадало с центром фотоприемной площадки соответствующего фотоэлемента. Для электрической изоляции задняя панель с установленными платами была покрыта ламинирующей пленкой.

Исследования проводились в лабораторных условиях при комнатной температуре, $\sim 22^{\circ} \mathrm{C}$. Незначительные

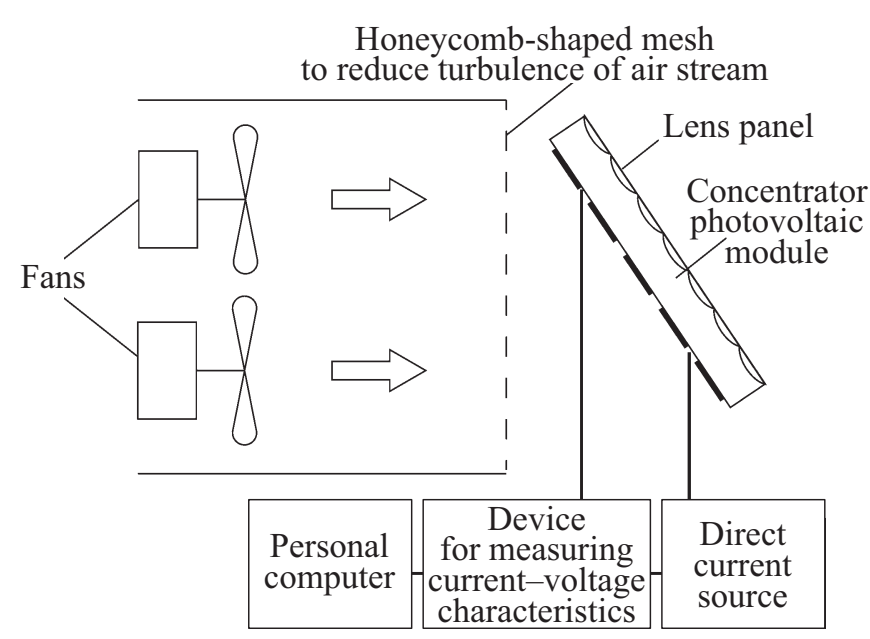

Рис. 1. Схема установки для оценки влияния скорости и направления воздушного потока на параметры, характеризующие тепловые свойства концентраторных модулей.

изменения температуры в процессе измерений были учтены путем внесения соответствующих поправок при расчетах температуры перегрева ФЭП.

Схема экспериментальной установки, которая была использована для исследований, показана на рис. 1. Генератор воздушного потока состоял из трубы прямоугольного сечения, гомогенизатора и двух вентиляторов диаметром $40 \mathrm{~cm}$, при этом была возможность регулировки скорости вращения. Установка позволяла имитировать скорости ветра от 0 до $3 \mathrm{M} / \mathrm{c}$ при неравномерности воздушного потока $\sim 15 \%$. Направление потока было выбрано горизонтальным, при этом его направление относительно модуля изменялось за счет изменения положения последнего.

Измерения тепловых параметров образцов были проведены при трех значениях скорости воздушного потока: 0,1 и $2 \mathrm{M} / \mathrm{c}$.

\section{3. Методика измерения теплового сопротивления системы „ФЭП-окружающая среда“}

Метод измерения теплового сопротивления состоит из двух этапов. На первом этапе при помощи термостата определяется связь между прямым падением напряжения и температурой исследуемого модуля. Для этого осуществляется запись вольт-амперной характеристики модуля при комнатной температуре. Затем исследуемый модуль помещается в термостат, внутри которого происходит нагрев модуля до определенной температуры. После экспозиции образца в термостате в течение времени, необходимого для равномерного нагрева всех его частей, осуществляется быстрая запись его ВАХ. Запись ВАХ проводится на переднем фронте импульса тока длительностью $0.15-0.2 \mathrm{мc}$, что позволяет получать значения напряжения, соответствующие 
температуре $p-n$-перехода. За данное время измерения температура ФЭП не успевает значительно измениться из-за достаточной теплоемкости ФЭП. Далее, сопоставляя значения прямого падения напряжения при разной температуре ФЭП, находим значения температурного коэффициента для различных значений тока.

На втором этапе при использовании полученных значений температурного коэффициента разница между измеренными значениями прямого падения напряжения для нагретого модуля и модуля, находящегося при комнатной температуре, может быть преобразована в разницу температур $p-n$-переходов ФЭП.

При исследовании параметров, характеризующих тепловые свойства модулей, нагрев последних осуществлялся за счет подвода электрической мощности при пропускания тока через фотоэлектрический модуль в прямом направлении. Вся подводимая электрическая мощность преобразовывалась в тепловую, величина которой определялась произведением величины силы постоянного тока, протекающего в прямом направлении, и падения напряжения на модуле. Данный подход позволял обеспечить контролируемый нагрев измеряемого модуля и имитировать процессы переноса и рассеивания тепла, возникающие при эксплуатации модуля на солнце. Необходимо отметить, что изменяя величину силы тока, пропускаемого в прямом направлении, можно имитировать условия, соответствующие разным режимам преобразования излучения и различным уровням освещенности. Тепловое равновесие между всеми частями модуля и окружающей средой достигалось за время, равное 30 мин. По истечении этого времени производилось быстрое отключение цепи подвода электрической мощности (длительность переключения составляет 0.15-0.2 мс), в течение которого записывалась ВАХ модуля.

При подведении тепловой мощности к фотоэлектрическому модулю температура перегрева $p-n$-переходов фотопреобразователей $\left(\Delta T_{p-n}\right)$ вычисляется по следующей формуле:

$$
\Delta T_{p-n}=\frac{U_{\mathrm{hot}}-U_{\mathrm{amb}}}{\beta_{\mathrm{mod}}}
$$

где $U_{\text {hot }}$ и $U_{\mathrm{amb}}$ - прямое падение напряжения для нагретого модуля и модуля находящегося при комнатной температуре соответственно, измеренные для одной и той же величины силы тока, $\beta_{\bmod }$ - температурный коэффициент для модуля, равный величине температурного коэффициента для одиночного ФЭП, умноженной на количество последовательно соединенных элементов.

Далее, зная значение подводимой тепловой мощности и соответствующую ей температуру перегрева ФЭП относительно окружающей среды, вычисляем величину теплового сопротивления системы „ФЭП-внешняя среда“:

$$
r_{\text {th }}=\Delta T_{p-n} S_{\text {lens }} / P_{\text {El }},
$$

где $S_{\text {lens }}$ - общая площадь линзовой панели модуля, $\Delta T_{p-n}-$ средняя температура перегрева ФЭП и $P_{\mathrm{El}}-$ подводимая тепловая мощность.

\section{4. Результаты}

На первом этапе исследований тепловых параметров модулей в термостате были проведены измерения прямых падений напряжения при различных температурах для двух модулей с теплосбрасывающими платами из меди и стали. Были проведены расчеты температурных коэффициентов для различных значений тока, которые представлены на рис. 2.

При исследовании величин разогрева ФЭП при подводе тепла за счет пропускания электрического тока в прямом направлении сначала были проведены измерения для модулей, расположенных под углом $45^{\circ}$ к горизонту, при отсутствии обдува. При такой конфигурации естественная конвекция воздуха играет основную роль в процессах переноса и рассеивания тепла. Рис. 3, a иллюстрирует эффект неравномерного нагрева задней стенки модуля в этом режиме за счет восходящих воздушных потоков, существующих как внутри, так и снаружи модуля.

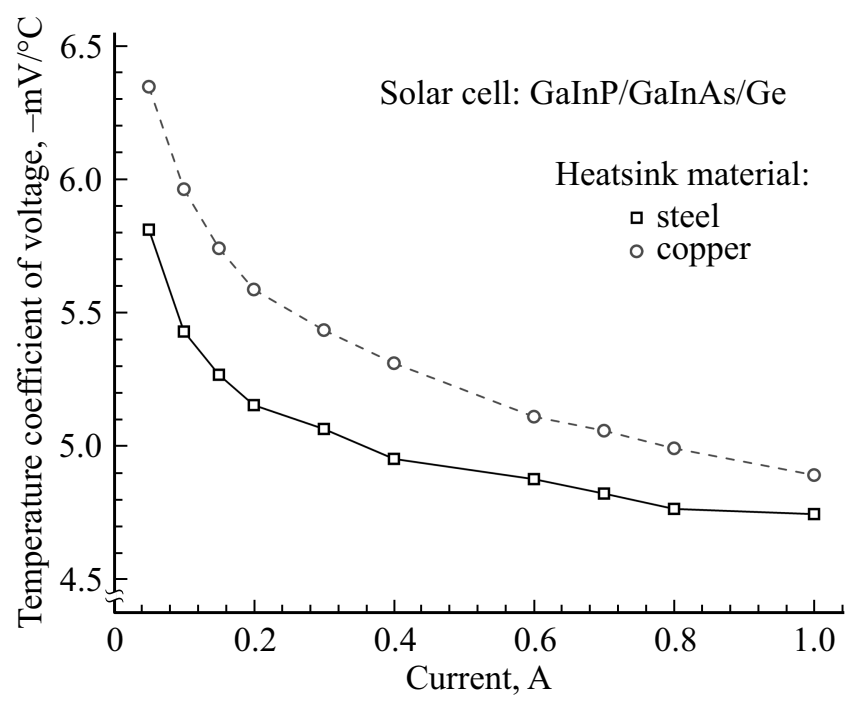

Рис. 2. Зависимость температурного коэффициента напряжения $(\beta)$ от величины тока, пропускаемого в прямом направлении, для концентраторных модулей с теплосбрасывающими платами из меди и стали.

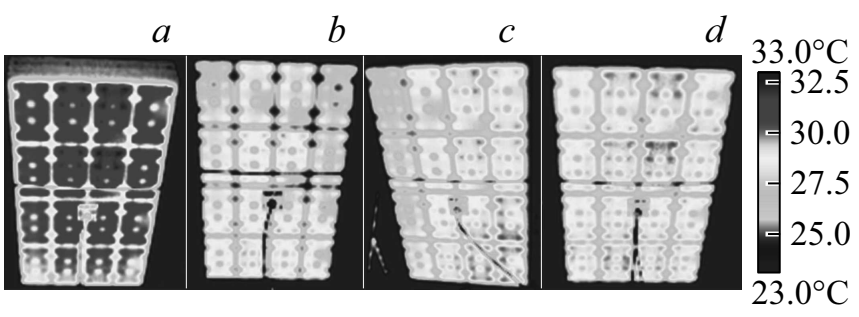

Рис. 3. Изображения в инфракрасном излучении задней фотоприемной панели модуля, полученные при помощи тепловизора. Подводимая электрическая мощность 50 Вт, условия обдува: $a-$ без обдува, $b-$ обдув со стороны теплоотводов, $c$ - обдув вдоль теплоотводов, $d-$ обдув со стороны линзовой панели. 


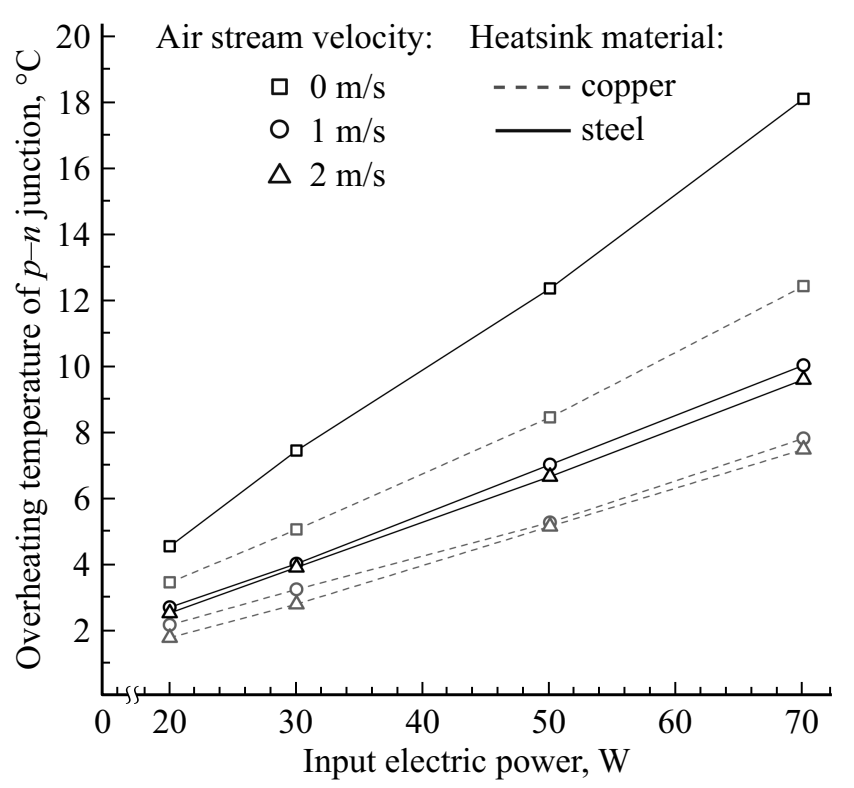

Рис. 4. Зависимости температуры перегрева ФЭП в концентраторном модуле от величины подводимой к нему электрической мощности для модулей с медными и стальными теплосбрасывающими платами при осуществлении обдува модуля со стороны задней стенки.

Неравномерность нагрева обусловливается в том числе и распределением протекающего тока через ФЭП модуля, неравномерность которого усиливается при увеличении разности температур между отдельными его элементами. Отметим, что при работе модуля в стандартных условиях на солнце этот эффект отсутствует за счет равномерного распределения падающей солнечной энергии по всей поверхности концентраторного модуля. Данный факт накладывает ограничения на выбор подводимой электрической мощности. С одной стороны, она должна быть достаточно большой для обеспечения существенного нагрева модуля и повышения точности определения температуры перегрева, с другой, - достаточно низкой, позволяющей нивелировать эффект неравномерного протекания тока через ФЭП и соответственно их неравномерный нагрев. Исходя из вышесказанного, максимальную подводимую электрическую мощность мы установили равной 70 Вт.

При наличии обдува температура задней стенки модуля значительно уменьшается и ее распределение становится более равномерным (рис. $3, b, c, d$ ). Направление воздушного потока почти не влияет на температуру модуля при обдуве со стороны теплоотводов.

Для модулей с медными и стальными теплосбрасывающими платами при различных условиях обдува были получены зависимости температуры перегрева $p-n$-перехода ФЭП от величины подводимой тепловой мощности (рис. 4). Зависимость температуры перегрева от подводимой мощности является линейной. Температура перегрева заметно уменьшается при обдуве модуля, однако при увеличении скорости воздушного потока выше $1 \mathrm{~m} / \mathrm{c}$ дальнейшего снижения температуры не происходит. Причиной этого может являться низкая теплопроводность ламинирующей пленки на задней панели модуля.

Для различных скоростей и направлений воздушного потока были рассчитаны значения теплового сопротивления системы „ФЭП-окружающая среда“ для модулей с медными и стальными теплосбрасывающими платами. Результаты расчетов приведены на рис. 5, $a$ для величины силы тока $0.6 \mathrm{~A}$, и на рис. $5, b$ - для тока $1 \mathrm{~A}$. Заметно небольшое различие в результатах, полученных при одной и той же подводимой тепловой мощности и температуре окружающей среды, но при различных значениях силы тока (рис. 5). Это объясняется незначительным нагревом ФЭП за время импульсных измерений BAX при больших величинах тока.
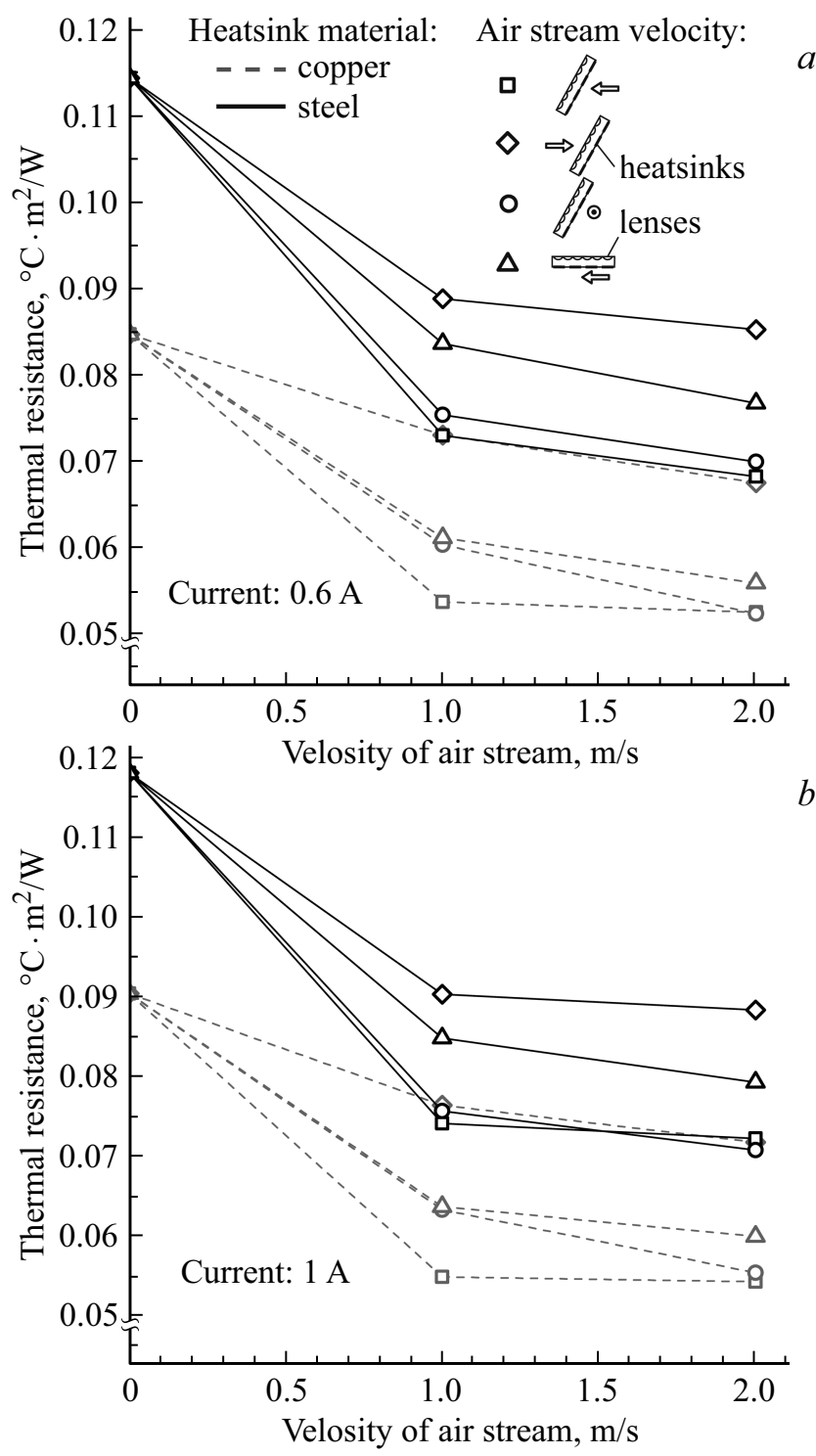

Рис. 5. Сравнение величин теплового сопротивления при различных условиях обдува для модулей с теплосбрасывающими платами из меди и стали. Подводимая электрическая мощность 50 Вт. 
В независимости от скорости воздушного потока и его направления, температура перегрева ФЭП, установленных на медных теплосбрасывающих платах, была на 35-40\% ниже температуры ФЭП, установленных на платы из стали. Однако несмотря на лучшие условия для рассеяния тепла в КФЭМ с медными теплосбрасывающими платами, сталь можно использовать для теплосбрасывающих плат из-за ее низкой стоимости и лучшего согласования коэффициентов теплового расширения стали, материала подложек фотоэлементов $(\mathrm{Ge})$ и стекла.

\section{5. Заключение}

Проведены исследования влияния условий обдува на величину перегрева ФЭП в концентраторных фотоэлектрических модулях. Для этого была использована лабораторная методика определения теплового сопротивления системы „ФЭП-окружающая среда“. Величина теплового сопротивления была рассчитана для различных положений модуля, скоростей и направлений обдува. Направление обдува не оказывает сильного влияния на температуру модуля за исключением случая, когда обдув осуществлялся со стороны линзовой панели. Увеличение скорости воздушного потока более величины $1 \mathrm{~m} / \mathrm{c}$ не приводит к заметному снижению температуры модуля вследствие низкой теплопроводности ламинирующей пленки, которая ограничивает эффективность теплосброса концентраторного модуля. Величина перегрева ФЭП, установленных на медных теплоотводящих платах, на $\sim 40 \%$ ниже перегрева ФЭП на стальных платах.

Исследование выполнено за счет гранта Российского научного фонда (проект № 17-79-30035).

\section{Список литературы}

[1] V.D. Rumyantsev. Concentrator Photovolt. SSOS, 130, 151 (2007).

[2] M. Muller, C. Deline, B. Marion, S. Kurtz, N. Bosco. AIP Conf. Proc., 1407, 331 (2011).

[3] V.D. Rumyantsev, A.V. Chekalin, N.Yu. Davidyuk, D.A. Malevskiy, P.V. Pokrovskiy, N.A. Sadchikov, A.N. Pan'chak. AIP Conf. Proc., 1556, 138 (2013).

[4] V.D. Rumyantsev, V.M. Andreev, A.V. Chekalin, N.Yu. Davidyuk, O.A. Im, E.V. Khazova, N.A. Sadchikov. AIP Conf. Proc., 1556, 185 (2013).

[5] V.D. Rumyantsev, A.V. Chekalin, N.Yu. Davidyuk, D.A. Malevskiy, M.Z. Shvarts, A. Luque, V.M. Andreev. AIP Conf. Proc., 1616, 154 (2014).

[6] V.D. Rumyantsev, A.V. Chekalin, N.Yu. Davidyuk, N.A. Sadchikov, A. Luque. Prog. Photovolt.: Res. Appl., 24 (2), 211 (2016).

[7] R. Núñez, I. Antón, S. Askins, G. Sala, K. Araki. AIP Conf. Proc., 1616, 144 (2014).

[8] V.D. Rumyantsev, A.V. Andreeva, A.V. Chekalin, N.Yu. Davidyuk, D.A. Malevskiy, P.V. Pokrovskiy, N.A. Sadchikov. Proc. 31st Eur. PV Solar Energy Conf. (2015) p. 1403.

\section{Influence of heat dissipation conditions on characteristics of concentrator photovoltaic modules}

\author{
A.V. Andreeva ${ }^{1}$, N.Yu. Davidyuk ${ }^{2}$, D.A. Malevskiy ${ }^{1}$, \\ A.N. Panchak ${ }^{1}$, N.A. Sadchikov ${ }^{1}$, A.V. Chekalin ${ }^{1}$ \\ ${ }^{1}$ loffe Institute, \\ 194021 St. Petersburg, Russia \\ 2 St. Petersburg Academic University, \\ 194021 St. Petersburg, Russia
}

\begin{abstract}
Influence of different heat dissipation conditions on temperature of solar cells and its distribution in concentrator photovoltaic modules were studied in the work. The modules based on Fresnel lenses and triple-junction GaInP/GaAs/Ge cells mounted on copper and steal heat sinks were investigated. During the indoor simulation of thermal processes occurring under the natural sun illumination, the elaborated method of measuring the temperature of the $p-n$-junctions was used. Obtained temperatures were used to calculate cell-to-ambient thermal resistance $r_{\text {th }}$. Forward current through the solar cells simulated thermal processes inside a concentrator photovoltaic module. The magnitude of forward voltage drop measured during a process of a fast $\mathrm{ON} / \mathrm{OFF}$ switching of a module was used to determine the temperature of $p-n$-junctions of solar cells. Heat dissipation conditions were varied using a wind tunnel.
\end{abstract}

